\title{
REVERSIBLE IMAGE HIDING USING SHIFTING OF MEAN VALUES WITH INTERLACED GROUPING AND SIDE GROUPING
}

\author{
Chef Chu \\ Department of Computer Science \\ National Tsing Hua University \\ Hsinchu, Taiwan \\ mr936309@cs.nthu.edu.tw
}

\author{
Long-Wen Chang \\ Department of Computer Science \\ National Tsing Hua University \\ Hsinchu, Taiwan \\ lchang@cs.nthu.edu.tw
}

\begin{abstract}
A new reversible data hiding is proposed. We group the pixels of block in the image into two groups with two methods called side grouping and interlaced grouping. Then, we calculate the mean values of the pixel values of the two groups, respectively. By using the relation between the difference of two mean values and the threshold value we embed secret data in the block. Because reversible data hiding may work in lossless environment, we use the multiple data hiding skill to embed secret data. By taking advantage of this skill, our algorithm could extract the secret data correctly and recover the cover image from the stego-image in a lossless environment. Also, we can extract secret data correctly in a low noisy environment due to JPEG compression.
\end{abstract}

\section{Categories and Subject Descriptors}

I.4.0 [Image Processing and computer vision]: General

\section{General Terms:}

Security

\section{Keywords \\ reversible data hiding, stego-image}

\section{INTRODUCTION}

Reversible data hiding [1] embeds a secret message into an image, and generate a stego-image. After extracting the secret message from the stego-image, it is possible to recover the original image. There are many applications in military, medical, and forensic images for reversible data hiding.

Zhicheng Ni et al. [2] proposed a lossless data hiding technique based on histogram modification. This algorithm uses the zero or minimum point, and the maximum point of the image histogram to embed secret data. The algorithm is very simple and the stego-

Mobimedia 2008 July 7-9, 2008, Oulu, Finland. Copyright 2008 ICST ISBN 978-963-9799-25-7/08/07 DOI 10.4108/ICST.MOBIMEDIA2008. 3918 image could get very high visual quality. Jun Tian proposed a reversible data hiding using difference expansion of pixel values $[3,4]$. After doubling the difference of two pixels, the least significant bit of the difference could embed a secret bit in it. This algorithm could implement with repetition so that the capacity is quite high. Vleeschouwer proposed an interesting reversible data hiding technique which uses the "center of mass" of the circle mapped from the histogram [5].

In this paper, we propose a new method by shifting the mean values of two groups of pixels values in a block of an image. The method can do reversible data hiding for lossless environment and also extract correct secrete data in a low noisy environment.

\section{THE PROPOSED ALGORITHM}

An image is divided into a sequence of blocks. The block size can be $2 \times 2,2 \times 4,4 \times 2,4 \times 4$, etc. If a block that is suitable to embed a data bit it is called embeddable; otherwise it is not embeddable. Each block is divided into two groups. The proposed reversible data hiding technique uses the concept of the mean value of a group in an embeddable block. The number of the embeddable blocks increases as the block size decreases. It is possible that the value of a pixel in the block may become overflow or underflow if it is shifted by a certain value to embed a data bit. We adopt the histogram modification to prevent overflow and underflow. The coordinate of the modified pixels for histogram modification is the side information that is needed for the receiver to recover the image. Because the number of the pixels needed to be modified is usually quite small, we can embed the side information into the image before embedding the secret data. Like Vleeschouwer's technique [5], our technique could resist against the JPEG compression by using a multiple bit hiding strategy.

The flowchart of embedding and extracting procedure are depicted in Figure 1 and Figure 2, respectively. They both include histogram modification. In the embedding process, we need to record the coordinates of the modified pixels as side information after histogram modification, and embed this side information together with the secret data into the pre-processed image. In the extracting phase, we can use this side information and histogram modification to recover the original one.

We also need to design a data format of secret data for extraction. The data format of the secret data is shown in Figure 3. The first bit $b_{1}$ means whether the image is pre-processed by 
histogram modification or not. If $b_{1}$ is 0 , there is no pixel needed to be shifted, the other bits from $b_{2} \sim b_{N}$ are secret data, where $\mathrm{N}$ is the number of the total bits of side information. If $b_{1}$ is 1 , the image has some modified pixels. $b_{2} \sim b_{7}$ record how many the modified pixels are, and $b_{8} \sim$ $b_{(16 n+7)}$ are the coordinates of the modified pixels, where $n$ is the decimal value of $b_{2} \sim b_{7}$. The rest are the secret data we want to embed.

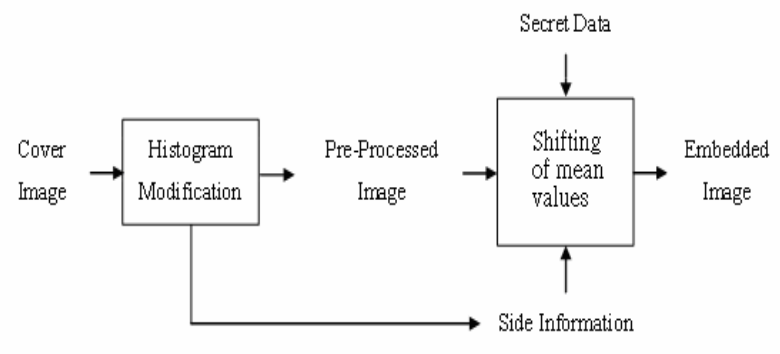

Figure 1: The embedding procedure

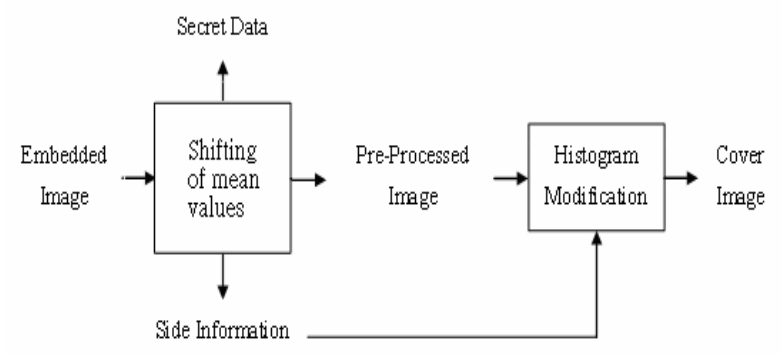

Figure 2: The extracting procedure

\begin{tabular}{|l|l|l|l|}
\hline$b_{1}$ & $b_{2} \sim b_{7}$ & $b_{8} \sim b_{(16 n+7)}$ & $b_{(16 n+8)} \sim b_{N}$ \\
\hline
\end{tabular}

Figure 3: The data format of embedded data

\subsection{Histogram Modification}

To prevent overflow and underflow, we use histogram modification before the shifting of mean value of a group in the coding block in the embedding phase.

For the pixels whose gray values are greater than 255- $T$, the gray values of these pixels need to be decrease by $T$ so that the gray values become equal to or less than $255-T$. For the pixels whose gray values less than $T$, the gray values need to be increased by $T$ so that the gray values become equal to or greater than $T$. The equation of histogram modification for a pixel $f(x, y)$ in an image is:

$$
f(x, y)=\left\{\begin{array}{cl}
f(x, y)-T & \text {,if } f(x, y)>255-T \\
f(x, y)+T & \text {,if } f(x, y)<T \\
f(x, y) & \text {,otherwise }
\end{array}\right.
$$

For a given image, we embed 1 bit in each embeddable block of the image. The block size could be $2 \times 2,2 \times 4,4 \times 2$, or $4 \times 4$. The block is divided into two groups, G1 and G2, as shown in Figure 4. The grouping methods are called side grouping and interlaced grouping.

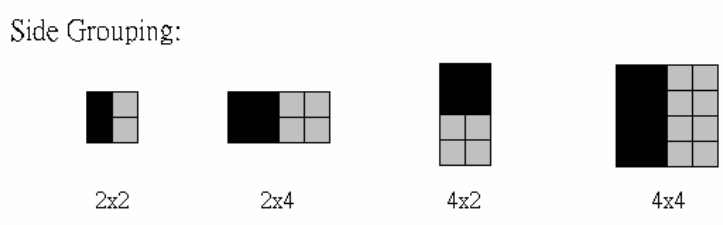

Interlaced Grouping:

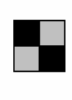

$2 \times 2$

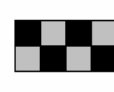

$2 \times 4$

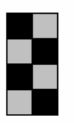

$4 \times 2$

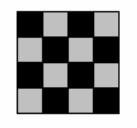

$4 \times 4$
Figure 4: 2 kinds of pixel grouping for 4 kinds of blocks

To explain the proposed reversible data hiding algorithm, the following notations are used.

$\mathrm{G}_{1}$ : The set of black pixels in a block in Figure 4

$\mathrm{G}_{2}$ : The set of gray pixels in a block in Figure 4

$\mathrm{P}_{1}$ : a pixel in $\mathrm{G}_{1}$

$\mathrm{P}_{2}$ : a pixel in $\mathrm{G}_{2}$

$\mathrm{F}\left(\mathrm{P}_{1}\right)$ is the pixel value of $\mathrm{P}_{1}$

$\mathrm{F}\left(\mathrm{P}_{2}\right)$ is the pixel value of $\mathrm{P}_{2}$

$\mathrm{M}_{1}$ : The mean of pixel values in $\mathrm{G}_{1}$

$\mathrm{M}_{2}$ : The mean of pixel values in $\mathrm{G}_{2}$

$\mathrm{T}$ : A threshold value

S: A bit we want to embed or extract

D: The difference of the two mean values, $M_{1}$ and $M_{2}$,

$\mathrm{D}=\mathrm{M}_{1}-\mathrm{M}_{2}$

After histogram modification, since $0<\mathrm{M}_{1}<$ (255$\mathrm{T})$ and $0<\mathrm{M}_{2}<(255-\mathrm{T})$ we have $(\mathrm{T}-255)<\mathrm{D}<$ $(255-\mathrm{T})$. Figure 5 shows the range of $\mathrm{D}$ after histogram modification. The black line means the difference value D before our watermark embedding algorithm. 


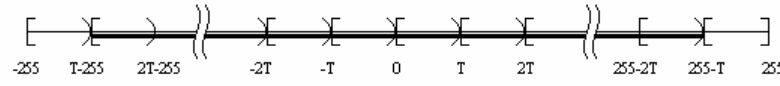

Figure 5 The range of difference value D of a block after histogram modification

When $-\mathrm{T}<\mathrm{D}<\mathrm{T}$, the block is embeddable; otherwise the block is not embeddable. The algorithm that embeds a bit is written as below. The ranges of $\mathrm{D}$ when embedding and extracting secret bits are depicted in Figure 6 and 7, respectively.

Algorithm that embed a bit:

If $(0 \leqq \mathrm{D}<\mathrm{T})$

$$
\text { If }(\mathrm{S}=0)
$$

$\mathrm{F}\left(\mathrm{P}_{1}\right)-\mathrm{T}$, for all pixel $\mathrm{P}_{1}$ in $\mathrm{G}_{1}$

$$
\text { Else ( } \mathrm{S}=1 \text { ) }
$$

$$
\mathrm{F}\left(\mathrm{P}_{1}\right)+\mathrm{T} \text {, for all pixel } \mathrm{P}_{1} \text { in } \mathrm{G}_{1}
$$

If $(-\mathrm{T} \leqq \mathrm{D}<0)$

$$
\text { If } \begin{aligned}
(\mathrm{S}= & 0) \\
& \mathrm{F}\left(\mathrm{P}_{1}\right)-\mathrm{T} \text {, for all pixel } \mathrm{P}_{1} \text { in } \mathrm{G}_{1}
\end{aligned}
$$

Else $(\mathrm{S}=1)$

$$
F\left(P_{1}\right)+T \text {, for all pixel } P_{1} \text { in } G_{1}
$$

If $(\mathrm{D} \geqq T)$

$\mathrm{S}$ is not embedded in the block, and

$\mathrm{F}\left(\mathrm{P}_{1}\right)+\mathrm{T}$, for all pixel $\mathrm{P}_{1}$ in $\mathrm{G}_{1}$

If $(\mathrm{D}<-\mathrm{T})$

$\mathrm{S}$ is not embedded in the block, and

$F\left(P_{1}\right)-T$, for all pixel $P_{1}$ in $G_{1}$

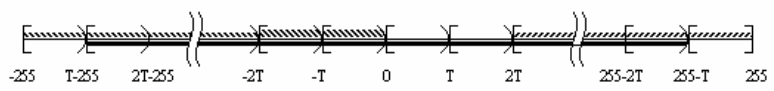

(a)

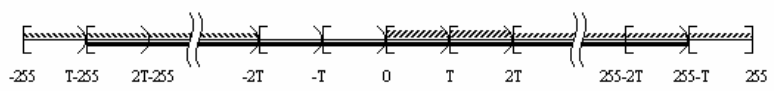

(b)
Figure 6 Shifting of D when (a) 0 is embedded (b) 1 is embedded

Figures 7(a) and 7(b) illustrate embedding 0 and 1, respectively. The black line means the difference value D before our algorithm, the slash $\%$ ' means the difference value after the mean value is added by $\mathrm{T}$ and the range of the backslash ' $'$ ' means the difference $D$ after the mean value is subtracted by T. Please also note that there are long slash area, short lash area, long backslash area and short backslash area. For example, in Figure 7(b), the long slash area is $[0, T)$, in Figure $7(\mathrm{a})$, the short slash area is $[2 \mathrm{~T}$, 255], the long backslash area is [-T, 0) and the short backslash area is $[-255,-2 \mathrm{~T})$. The short slash area and the short backslash area mean that the pixel value whose mean value is in this area is shifted by $\mathrm{T}$ but is not embedded a secret bit, and the long slash area and long backslash area mean that the pixel whose mean value is in this area is shifted by $\mathrm{T}$ and is embedded a secret bit.

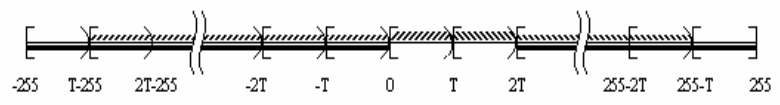

(a)

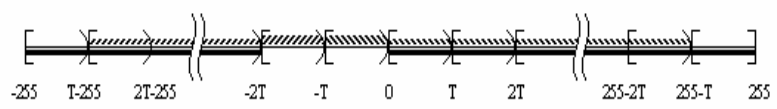

(b)

Figure 7 Shifting of D when the secret bit (a) 0 is extracted (b) 1 is extracted.

\section{SIMULATION RESULTS}

Two standard test images, Lena and Baboon, are used to be experimented by our proposed technique. We show our experimental images of $256 \times 256$ Lena in Figure 8 using the side grouping method with $2 \times 2$ block size and different thresholds. Secondly, we show the experimental results of $256 \times 256$ Baboon in Figure 9 using the interlaced grouping method with $2 \times 2$ block size and different thresholds. Table 1 shows PSNRs for various thresholds. Table 2 shows capacity and bitrate of Lena using interlaced grouping method with different thresholds and block sizes. Table 3 shows capacity and bitrate of Lena using the side grouping methods with different thresholds and block sizes. From these three tables, when the threshold becomes big, the embedding capacity becomes large. Table 4 shows the correct bits extracted from JPEG images with different quality factors using side grouping and $2 \times 4,4 \times 4$, and $4 \times 8$ 
block sizes. It shows that the correct bits extracted from JPEG images with different quality factors using side grouping and $2 \times 4,4 \times 4$, and $4 \times 8$ block sizes

\section{CONCLUSIONS}

We proposed a reversible data hiding technique based on side grouping and interlaced grouping. Our technique uses the mean value other than the center of mass, in order to decrease the computational complexity. As to capacity, because the neighboring values usually are similar, the mean values could be very close with small block size. Small size increases the total number of embeddable blocks and leads to a high capacity. Our technique could get high PSNR values in the data hided images because only half of pixels in a block are changed other than total of pixels. Also, we can extract secret data correctly in a low noisy environment due to JPEG compression.

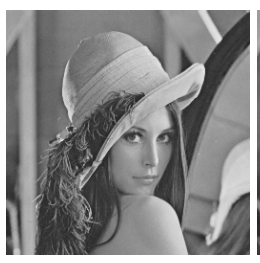

(a)

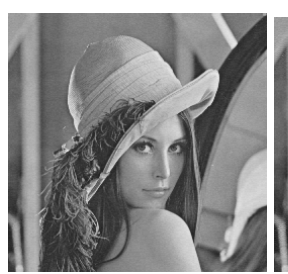

(d)

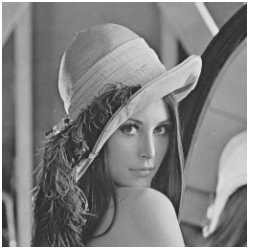

(b)

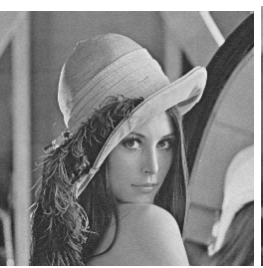

(e)

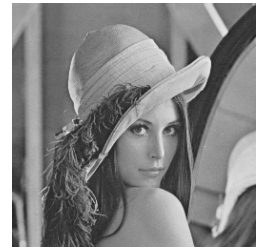

(c)

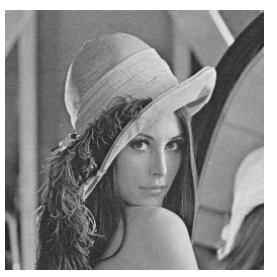

(f)
Figure 8:(a) Original 256×256 gray leve limage (b) $\mathrm{T}=1$

(c) $\mathrm{T}=2(\mathrm{~d}) \mathrm{T}=3(\mathrm{e}) \mathrm{T}=4$ (f) $\mathrm{T}=5$

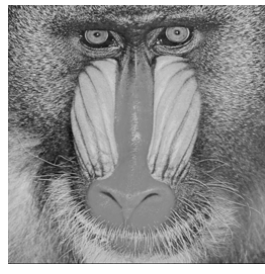

(a)

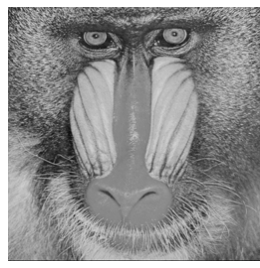

(b)

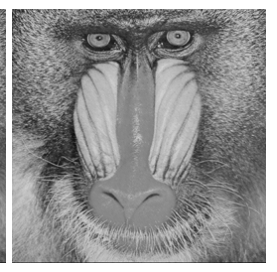

(c)

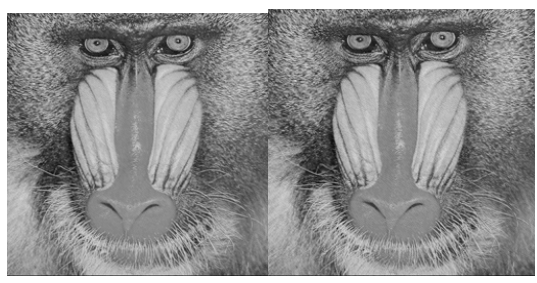

(d)

(e)

(f)

Figure 9: (a) Original 256×256 gray level image (b) $\mathrm{T}=1$ (c) $\mathrm{T}=2(\mathrm{~d}) \mathrm{T}=3(\mathrm{e}) \mathrm{T}=4$ (f) $\mathrm{T}=5$

\begin{tabular}{|c|c|c|c|c|c|}
\hline Threshold & 1 & 2 & 3 & 4 & 5 \\
\hline PSNR & 51.1411 & 45.1205 & 41.5987 & 39.0999 & 37.1617 \\
\hline
\end{tabular}

Table 1: PSNR of stego-image with different thresholds

\begin{tabular}{|c|c|c|c|c|c|c|}
\hline \multicolumn{2}{|c|}{ Theck } & 1 & 2 & 3 & 4 & 5 \\
\hline \multirow{2}{*}{$2 \times 2$} & Capacity & 4635 & 8172 & 10539 & 12064 & 12961 \\
\cline { 2 - 7 } & Bitrate & 0.0707 & 0.1247 & 0.1608 & 0.1841 & 0.1978 \\
\hline \multirow{2}{*}{$2 \times 4$} & Capacity & 3039 & 5021 & 6158 & 6684 & 6998 \\
\cline { 2 - 7 } & Bitrate & 0.0464 & 0.0766 & 0.0937 & 0.1020 & 0.1068 \\
\hline \multirow{2}{*}{$4 \times 2$} & Capacity & 3085 & 5051 & 6084 & 6684 & 7011 \\
\cline { 2 - 7 } & Bitrate & 0.0467 & 0.0771 & 0.0928 & 0.1020 & 0.0170 \\
\hline \multirow{2}{*}{$4 \times 4$} & Capacity & 1085 & 1959 & 2563 & 2981 & 3232 \\
\cline { 2 - 7 } & Bitrate & 0.0166 & 0.0298 & 0.0391 & 0.0455 & 0.0493 \\
\hline
\end{tabular}

Table 2: Capacity and bitrate of Lena using interlaced grouping method with different thresholds and block sizes

\begin{tabular}{|c|c|c|c|c|c|c|}
\hline \multicolumn{2}{|c|}{$x$} & 1 & 2 & 3 & 4 & 5 \\
\hline \multirow{2}{*}{$2 \times 2$} & Capacity & 3358 & 7051 & 9397 & 10962 & 12004 \\
\hline & Bitrate & 0.0589 & 0.1076 & 0.1434 & 0.1673 & 0.1832 \\
\hline \multirow{2}{*}{$2 \times 4$} & Capacity & 1202 & 2267 & 3154 & 3780 & 4265 \\
\hline & Bitrate & 0.0183 & 0.0346 & 0.0481 & 0.0577 & 0.0651 \\
\hline \multirow{2}{*}{$4 \times 2$} & Capacity & 1776 & 3180 & 4241 & 4956 & 5426 \\
\hline & Bitrate & 0.0269 & 0.0485 & 0.0647 & 0.0756 & 0.0828 \\
\hline \multirow{2}{*}{$4 \times 4$} & Capacity & 345 & 670 & 1002 & 1280 & 1506 \\
\hline & Bitrate & 0.0053 & 0.0102 & 0.0153 & 0.0195 & 0.0230 \\
\hline
\end{tabular}


Table 3: Capacity and bitrate of Lena using side grouping methods with different thresholds and block sizes

\begin{tabular}{|c|c|c|c|c|c|c|c|c|c|}
\hline OF & 90 & 80 & 70 & 60 & 50 & 40 & 30 & 25 & 20 \\
\hline $\begin{array}{c}\text { slock } \\
\text { size }\end{array}$ & 100 & 100 & 100 & 97 & 87 & 81 & 79 & 67 & 59 \\
\hline $4 \times 2$ & 100 & 100 & 100 & 96 & 95 & 84 & 62 & 54 & 46 \\
\hline $4 \times 4$ & 100 & 100 & 100 & 100 & 100 & 98 & 88 & 83 & 64 \\
\hline
\end{tabular}

Table 4: The correct bits extracted from JPEG images with different quality factors using side grouping and $2 \times 4,4 \times 4$, and $4 \times 8$ block sizes

\section{REFERENCES}

[1] Yun Q. Shi, Zhicheng Ni, Dekun Zou, Changyin Liang and Guorong Xuan, "Lossless data hiding: Fundamentals, Algorithms and Applications," Proceeding of ISCAS 2004, Vol. 2, pp.23-26.

[2] Zhicheng Ni, Yun Q. Shi, Nirwan Ansari and Wei Su, "Reversible Data Hiding," IEEE Transactions on Circuits and Systems for Video Technology, Vollume 16, March 2006, pp. 354-362.

[3] Jun Tian, "High Capacity Reversible Data Embedding and Content Authentication," Proceeding of ICASSP 2003, Vol. 3, pp. $517-520$

[4] Jun Tian, "Reversible Data Embedding Using a Difference Expansion," IEEE Transactions on Circuits and Systems for video Technology, Vol.13, no.8, August 2003, pp. 890-896.

[5] Christophe De Vleeschouwer, Jean-François Delaigle, and Benoît Macq, "Circular Interpretation of Bijective Transformation in Lossless Watermarking for Media Asset Management," IEEE Transactions on Multimedia, Vol.5, no.1, March 2003, pp. 97-105. 